\title{
Biosynthesis of silver Nanoparticles from marine sponge Callysspongia diffusa associated - P. fluorescens BCPBMS-1
}

\section{Vasanthabharathi, V. Kalaiselvi and S. Jayalakshmi}

CAS in Marine Biology, Faculty of Marine Sciences Annamalai University, Parangipettai, Tamilnadu, India. Corresponding author bharathigene@rediffmail.com

\section{ABSTRACT}

New applications of nanomaterials are rapidly emerging. The synthesis of nanoparticles is a cornerstone of nanotechnology. Microbial cells are highlyorganized units, regarding morphology and metabolic pathways, capable of synthesizing well size-calibrated and well-structured particles. Furthermore, biogenic nanoparticles often are water-soluble and biocompatible, which is essential for many applications. Molecular identification of a novel strain P. fluorescens BCPBMS-1 from sponge Callysspongia diffusa (Mandapam Coast) through 16s rRNA ribotyping (Gen bank accession number: HQ907732). The silver nanoparticles were analyzed by UV-Visible spectroscopy. Their chemical composition was determined by FT-IR spectroscopy. SEM observationrevealed that silver nano particles are having spherical shape. The antibacterial activities of silver nanoparticles were screened against common human pathogen Escherichia coli, Proteus mirabilis, Salmonella typhi, Salmonella paratyphi, Vibrio cholerae, Klebsiella oxytoca, Klebsiella pneumoniae and Staphylococcus aureus. Among these $5 \mathrm{~mm}$ antibacterial activity was observed with E. coli, $4 \mathrm{~mm}$ with $P$. mirabilis and $S$. typhi, $3 \mathrm{~mm}$ activity was observed with $S$. paratyphi. These results suggest that Ag nanoparticles can be used as effective growth inhibitors in various microorganisms, making them applicable to diverse medical devices and antimicrobial control systems.

Keywords: Silver nanoparticles, FT-IR, SEM.

\section{INTRODUCTION}

Marine microbial biotechnology has opened up unexpected new horizons for finding novel organism for trapping their potential resources .Oceans account for more than $70 \%$ of the earth's surface and the microorganisms growing in marine environments are metabolically and physiologically diverse from the terrestrial organisms (Takizawa et al., 1993).

Ag-NPs (silver nanoparticles) are used in hygienic products including water Purification systems, linings of washing machine, dishwashers, refrigerators, and toilet seats (Rai et al., 2009) Thus, silver ions have been used in many kinds of formulations (Sondi \& Salopek-Sondi, 2004), and recently it was shown that hybrids of silver nanoparticles with amphiphilic hyper branched macromolecules exhibit effective antimicrobial surface coating (Ymonier et al., 2002). One of the most studied aspects of nanotechnology nowadays is their ability to offer the opportunity to fight microbial infections via synthesis of nanoparticles. The mechanism of prevention of bacterial growth by antibiotics is quite different from the mechanisms by which nanoparticles inhibit microbial growth. Therefore, nanoparticles have the potential to serve as an alternative to antibiotics and to control microbial infections. 
Chemical synthesis of nanoparticles leads to presence of traces of toxic chemical adsorbed on the surface which is undesirable in the medical applications of nanoparticles. Biosynthesis of silver nanoparticles provides an alternative to chemical and physical methods as it is cost effective, environment friendly, and it does not involve use of high pressure, energy, temperature and toxic chemicals. Currently, there is a growing need to development of environmentally safe processes for synthesis of nanoparticle that do not use toxic chemicals in the synthesis protocol. The microorganisms such as bacteria, yeast and now fungi play an important role in remediation of toxic metals through reduction of the metal ions, this was considered interesting as nanofactories very recently (Fortin \& Beveridge, 2000).

The biosynthesis of silver nanoparticles of different sizes, ranging from 1$70 \mathrm{~nm}$, and shapes, including spherical, triangular and hexagonal has been conducted using bacteria, fungi, plant extracts. The mechanisms for the bioreduction of silver by bacteria involve reducing and other proteins in which sulphur and carboxylate group from cell wall (Sathyavathi et al., 2010; Bhainsa \& De'Souza, 2006).

Various salts of silver and their derivatives are used as antimicrobial agents (Russell \& Hugo, 1994). Recent studies have reported that nanosized silver particles exhibit antimicrobial properties (Petica et al., 2008) Silver has been recognized to have inhibitory action on microbes (Rai et al., 2009).

\section{MATERIALS AND METHODS}

\section{Isolation of Pseudomonas sp. from the marine sponge Callyspongia diffusa}

The sponge was collected from Mandapam. It is situated $\left(79^{\circ} 8^{\prime} \mathrm{E}, 9^{\circ} 17^{\prime} \mathrm{N}\right)$ on a narrow tongue of land projecting from the southern part of the east coast of india.To the north of this penisular extension is in the Palk Bay and to the south the Gulf of Mannar.

The sponge sample soon after collection was transferred to a sterile polyethylene bag and transported at $4^{\circ} \mathrm{C}$ to the laboratory for the isolation of associated microbes. On reaching the laboratory, the invertebrate was brought to room temperature and cut aseptically into small pieces $(2 \times 2 \mathrm{~cm})$ using a sterile scissors. The pieces were freed from adhering particles by vortexing twice for $20 \mathrm{sec}$. with $2 \mathrm{ml}$ of sterile seawater. The seawater was decanted, which was once again replaced with sterile seawater with continued vortexing between washings. Finally, sample in sterile seawater was homogenized using sterilized mortar and pestle in a Laminar flow chamber. The homogenate was serially diluted up to $10^{-6}$ dilutions and then spread plated on King b agar (Peptone 20.0g, Dipotassium hydrogen phosphate $1.5 \mathrm{~g}$, Magnesium sulfate $1.5 \mathrm{~g}$, Agar $10.0 \mathrm{~g}, 50 \%$ Aged Sea water- $1000 \mathrm{ml}, \mathrm{pH} 7.2+/-0.2$ ). The plates were incubated at room temperature for $24-48 \mathrm{hrs}$.

\section{Identification of Pseudomonas sp by $16 S$ r RNA partial sequencing}

The genomic DNA extracted from the marine sponge associated Pseudomonas sp was PCR amplified for 16S rRNA genes using the universal bacterial primers Eubac27F (5'- AGA GTT TGA TCG TGG CTC AG- 3') and 1492R (5'- GGT TAC CTT GTT ACG ACT T-3'). This primer combination amplified a 1500bp 16S rDNA fragment.

The PCR product was purified using the QIAGEN PCR purification kit for Sequencing and further analysis. The partial 16S r RNA gene sequencing was done using Perkin Elmer applied biosystems and ABI Prism software was used to allign the sequence and compared sequences were retrieved by the queries generated by BLAST 
of Gen Bank Database. Phylogenetic analysis was performed with the MEGA 4.0 program (Molecular Evolutionary Genetics Analysis, Version 4.0).

\section{UV-Vis Spectra analysis}

About $50 \mathrm{~mL}$ aqueous solution of $1 \mathrm{mM}$ silver nitrate (AgNO3) was treated with $50 \mathrm{~mL}$ of P.fluorescens supernatant solution in a $250 \mathrm{~mL}$ Erlenmeyer flask $(\mathrm{pH}$ adjusted to 8.5). The whole mixture was put into a shaker at $40^{\circ} \mathrm{C}(200 \mathrm{rpm})$ for 5 days and maintained in the dark. Control experiments were conducted with un inoculated media, to check for the role of bacteria in the synthesis of nanoparticles. The reduction of $\mathrm{Ag}^{+}$ions was monitored by measuring the UV-Vis spectrum of the reaction medium after $72 \mathrm{hrs}$. UV-Vis spectral analysis was done by using UV-Vis spectrophotometer UV-2450 (Shimadzu). The nanoparticles were separated from the reaction mixture by centrifugation at $10,000 \mathrm{rpm}$ for 10 minutes at $4^{\circ} \mathrm{C}$ (Saifuddin et al., 2009).

\section{FT-IR analysis of silver nanoparticles}

The supernatant containing silver nanoparticles was lyophilized. It was subjected to FTIR analysis. The interaction between protein and silver nanoparticles were analysed by Fourier transform infrared analyzer (Perklin Elmer).

\section{SEM analysis of silver Nanoparticles}

The lyophilized samples were for SEM. Scanning Electron Microscopic (SEM) analysis was done by using JEOL JSM- 6360 SEM machine.

\section{Antibacterial activity of silver Nanoparticles}

Antibacterial assay was done by an agar-well diffusion method in aerobic condition. Bacterial pathogens such as Escherichia coli, Proteus mirabilis, Salmonella typhi, Salmonella paratyphi, Vibrio cholerae, Klebsiella oxytoca, Klebsiella pneumoniae and Staphylococcus aureus were spreaded on Muller Hinton agar plates. Then wells were made and $50 \mu \mathrm{l}$ of silver nanoparticles was inoculated. Antagonistic activity was detected after an incubation of $24-48 \mathrm{hrs}$ at $35^{\circ} \mathrm{C}$. The presence of zone of clearance on agar plates was used as an indicator for the antibacterial activity. $50 \mu 1$ of distilled water was used as control.

\section{RESULTS}

In the present study Phylogenetic tree revealed that $P$. fluorescens BCPBMS-1 (bioactive compound producing bacteria) which was isolated from marine sponge Callyspongia diffusa was closely related to P. fluorescens CIAH-Pf-196-16s strain (Fig. 1).

The tree Topologies were evaluated by bootstrap analyses based on 1,000 replicates and phylogenetic trees were inferred using the neighbour-joining method and submitted to NCBI Gen Bank (accession number: 1428145 HQ907732).

The appearance of a brown colour in solution containing the supernatant is a clear indication of the formation of silver nanoparticles in the reaction mixture (Fig. 2). The colour of the solution is due to the excitation of surface Plasmon vibrations (essentially the vibration of the group conduction electrons) in the silver nanoparticles. The colour intensity of the cell filtrate with $\mathrm{AgNO}_{3}$ was sustained even after 5 days incubation, which indicated that the particles were well dispersed in the solution, and there was no obvious aggregation. 


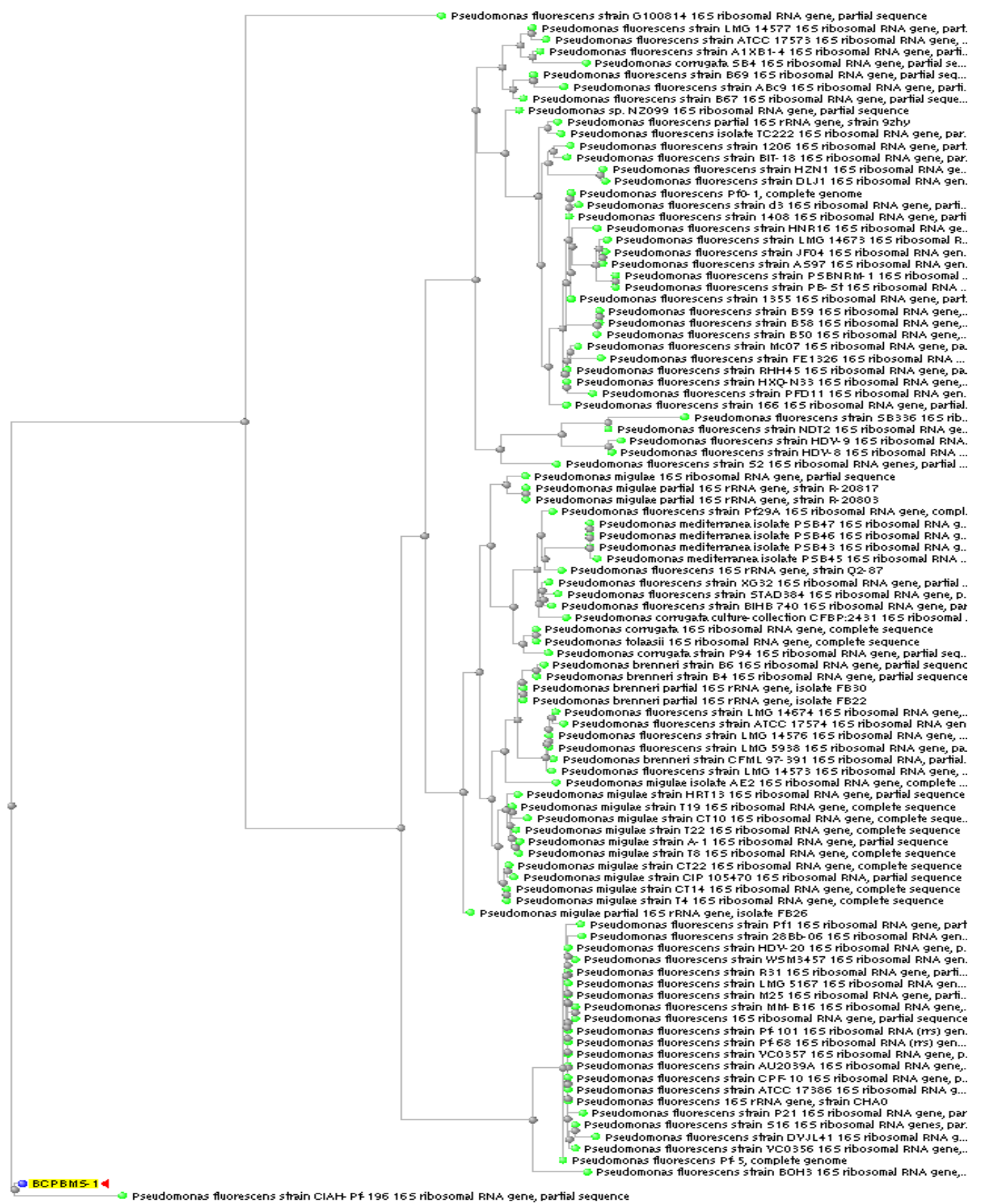

Fig.1: Phylogenetic tree view

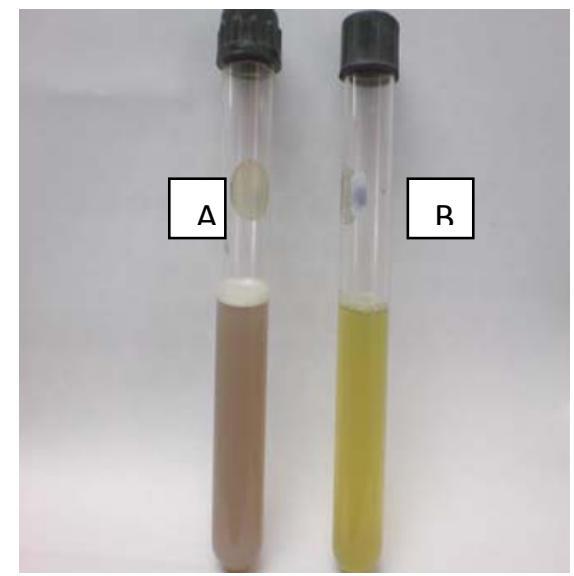

Fig. 2: P. fluorescens BCPBMS-1 supernatant after (A) and before (B) exposure to $\mathrm{Ag}^{+}$ions 
The spectra recorded from the $P$. fluorescens BCPBMS-1 reaction vessel at different wavelength were reported. The strong surface Plasmon resonance centered at ca. 350-400 $\mathrm{nm}$. Increased in the peak was observed at $400 \mathrm{~nm}$. The spectra clearly show the increase in intensity of silver solution indicating the formation of silver nanoparticles in the solution.

FT-IR is a powerful tool for identifying types of chemical bonds in a molecule by producing an infrared absorption spectrum that is like a molecular "fingerprint". FT-IR analysis was carried out to identify the possible bio-molecules and cell-metal ions interaction responsible for formation and stabilization of silver nanoparticles.

In the present investigation strong stretching bonds $\mathrm{O}-\mathrm{H}$ occurred $3434 \mathrm{~cm}^{-1}$. There is a very strong $\mathrm{C}-\mathrm{H}$ stretching vibration occurred at the wave number 2958 $\mathrm{cm}^{-1}$. The strong medium Methyl group has occurred at $2923 \mathrm{~cm}^{-1}$ and $2852 \mathrm{~cm}^{-1}$. The medium strong $2427 \mathrm{~cm}^{-1}$ shows the $\mathrm{O}-\mathrm{H}$. The wave number $2362 \mathrm{~cm}^{-1}, 2344 \mathrm{~cm}^{-1}$ $2092 \mathrm{~cm}^{-1}$ and $1717 \mathrm{~cm}^{-1}$ shows the $\mathrm{C}=\mathrm{O}$ stretching vibration. The medium stretching $\mathrm{N}-\mathrm{H}$ band has occurred at $1654 \mathrm{~cm}^{-1}, 1647 \mathrm{~cm}^{-1}, 1630 \mathrm{~cm}^{-1} .1637 \mathrm{~cm}^{-1}$ showed $\mathrm{C}=\mathrm{O}$ carboxyl group and $-\mathrm{C}=\mathrm{C}$ stretching $.1458 \mathrm{~cm}^{-1}$ shows amine and amino methyl stretching. The wave number $1438 \mathrm{~cm}^{-1}, 1384 \mathrm{~cm}^{-1}$ showed the medium strong C-N stretching. A medium strong stretching vibration occurred at the wave number 1245 $\mathrm{cm}^{-1}$. A medium weak stretching occurred at the wave number $1082 \mathrm{~cm}^{-1}$. A medium strong stretching C-O band has occurred at $919 \mathrm{~cm}^{-1}$. The wave number $555 \mathrm{~cm}^{-1}$ showed the strong medium rocking vibration represents $\mathrm{C}-\mathrm{Cl}$ bond (Fig. 3).

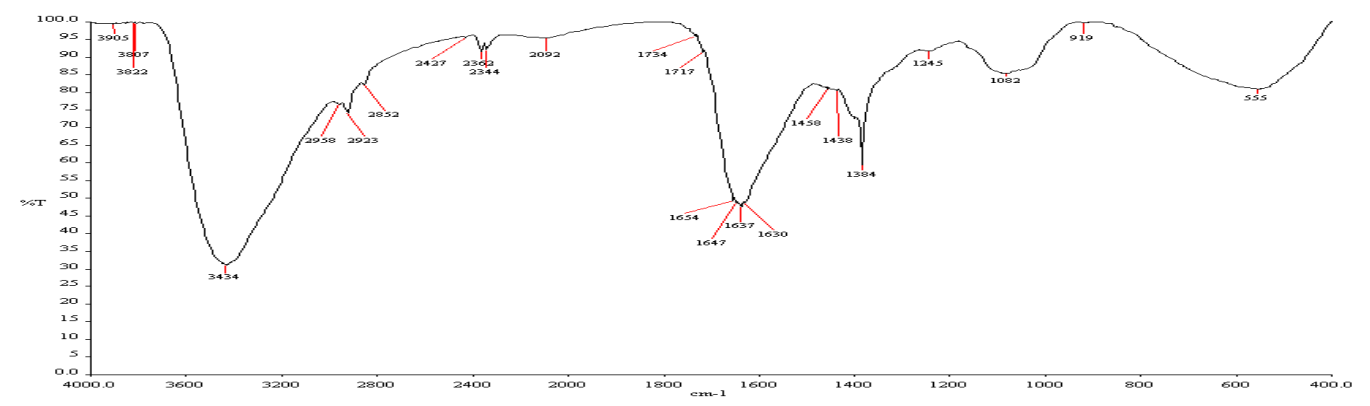

Fig. 3: FT-IR analysis of silver nanoparticles from the $P$. fluorescens BCPBMS-1

This SEM micrograph showed that $10 \mu \mathrm{m}$ size of nanoparticles synthesized from P. fluorescens BCPBMS-1 (Fig.4). The SEM micrographs of nanoparticle obtained in the filtrate showed that Ag-NPs are spherical shaped, well distributed without aggregation in solution.

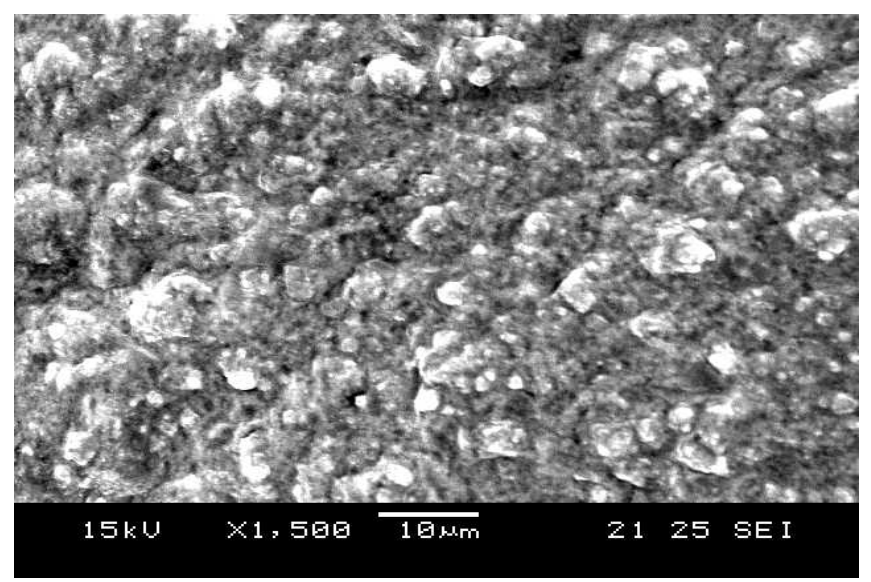

Fig. 4: SEM micrograph of silver nanoparticles 
In the present investigation among the tested pathogens antibactreial activity was observed with E. coli , P. mirabilis, S. typhi, S. paratyphi,. P. mirabilis. Whereas it was absence with $S$. aureus and $V$. cholera. Among these $5 \mathrm{~mm}$ antibacterial activity was observed with E. coli, $4 \mathrm{~mm}$ with $P$. mirabilis and S. typhi, $3 \mathrm{~mm}$ activity was observed with $S$. paratyphi (Figs. 5,6 \&7).

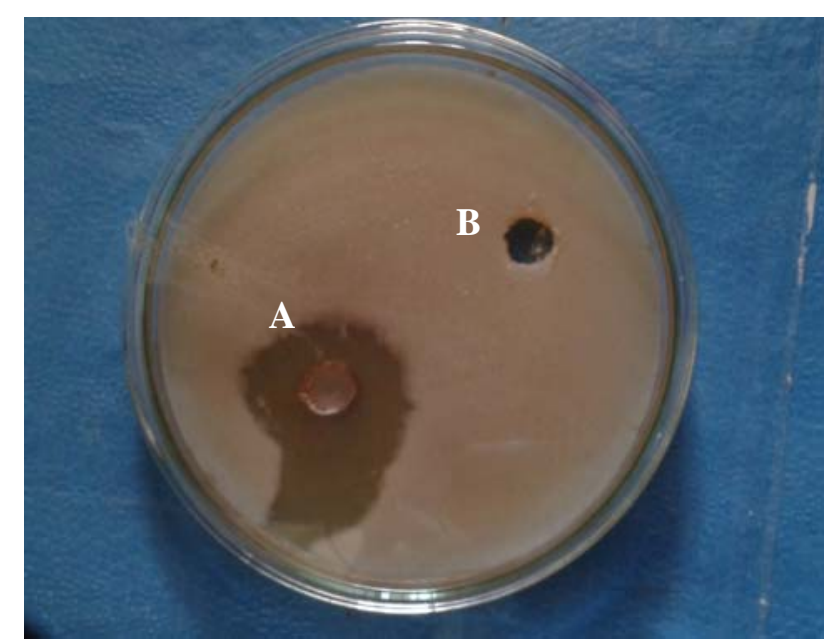

Fig.5 : Antibacterial activity of E.coli (A) and control (B).

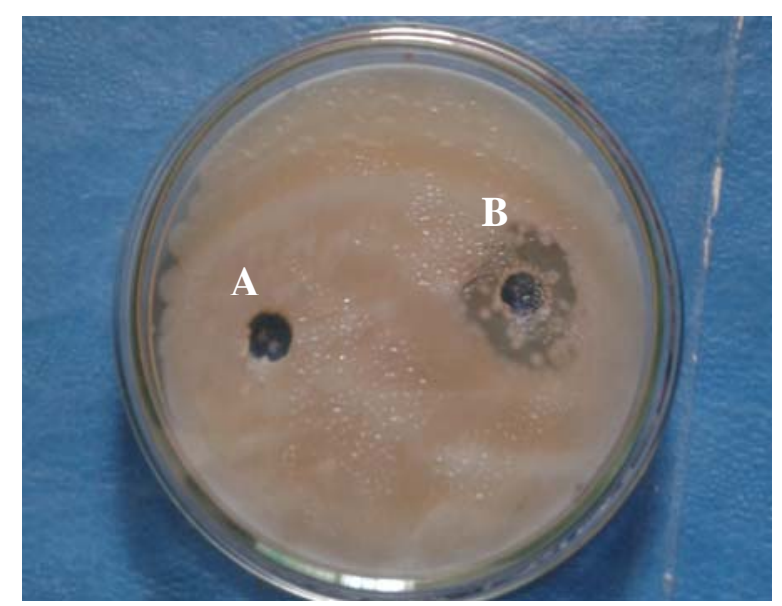

Fig. 6: Antibacterial activity of P. mirabilis (A) and control (B).

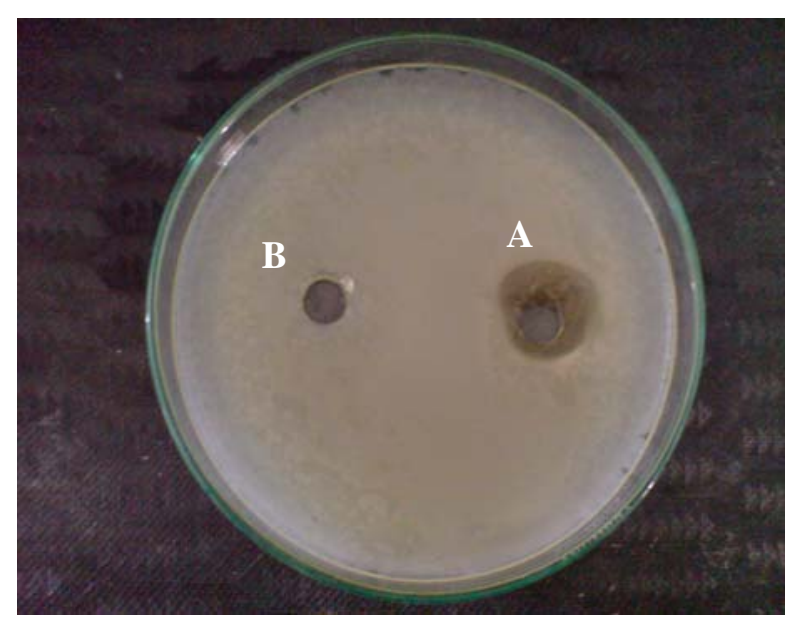

Fig. 7: Antibacterial activity of S. paratyphi (A) and control (B). 


\section{DISCUSSION}

The synthesis of nanoparticles is in the lime light in modern nanotechnology. The development of biologically inspired experimental processes for the synthesis of nanoparticles is evolving in to an important branch of nanotechnology. Compared with the traditional synthetic methods, biological systems provide a novel idea for the production of nano-materials (Bansal et al., 2011). Up to now, several microorganisms from bacteria to fungi have been reported to synthesize inorganic materials either intra- or extra cellularly and thus to be potentially utilized as ecofriendly nanofactories (Shankar et al., 2004; Mohanpuria et al., 2008).

Pseudomonas stutzeri AG259, isolated from silver mines, has been shown to produce silver nanoparticles (Klaus et al., 1999) and the bioreduction of Ag was also reported in Bacillus licheniformis. Vijayaraghavan et al. (2012), isolated silver nano particles from marine B. subtilis. Several reports on the biosynthesis of AgNPs using fungi, including F. acuminatum (Ip et al., 2006) and Penicillium fellutanum (Kathiresan et al., 2009). Huang et al. (2008) observed that the supernatant of $A$. terreus showed the ultraviolet-visible spectra with $\mathrm{AgNO}_{3}$ showed a strong broad peak at $440 \mathrm{~nm}$ (SPR band) which indicated the presence of AgNPs.

A similar observation has been made in P. aeruginosa (Jeevan et al., 2012) the wave number $1387 \mathrm{~cm}^{-1} \mathrm{C}-\mathrm{N}$ stretching, $1457 \mathrm{~cm}^{-1}$ amino and amino methyl stretching, $1639 \mathrm{~cm}^{-1} \mathrm{C}=\mathrm{O}$ carboxyl group and $\mathrm{C}=\mathrm{C}$ stretching. The present observation also correlate with Mubark Ali et al. (2011) from marine algae Oscillatoria willei observed N-H bond at $1651 \mathrm{~cm}^{-1}$.

Vivek et al. (2011) observed that the SEM analysis of silver nanoparticles synthesized by the help of Gelidiella acerosa extract having average mean size of the silver nanoparticles and seems to be spherical in morphology. Ganesh Babu et al. (2011) also observed the presence of silver nano particles in the cells of $B$. cereus ATCC by using SEM analysis.

Records show that Hippocrates recognized the role of silver in the prevention of disease and existing accounts suggest that the Romans stored wine in silver vessels to prevent spoilage. However, it is only in the last few decades that the mode of action of silver as an antimicrobial agent has been studied with any rigor.

Devina Merin et al. (2010) observed antibacterial activity of silver nano particles from marine microalgae against Klebsiella sp., Proteus vulgaris, Pseudomonas aeruginosa.

Selvakumar et al. (2012) observed significant antibacterial activity against $S$. aureus and $P$. aeruginosa by silver nano particles from marine derived Streptomyces rochei. In another study antibacterial activity of the bio-nanoparticles derived from Streptomyces sp. active against $S$. typhi, S. epidermidis, S. aureus, P. erogenosa, $P$. vulgaris and E. coli (Shirley et al ., 2010).

The present study suggests that silvernanoparticles can be used as effective growth inhibitors in various microorganisms, making them applicable to diverse medical devices and antimicrobial control systems.

\section{REFERENCES}

Bansal, V, Ramanathan, R. and Bhargava, SK. (2011). Fungus-mediated biological approaches towards "green" synthesis of oxide nanomaterials. Australian Journal of Chemistry. 64: 279-293. 
Bhainsa K.C., De'Souza, K. (2006). The role of Anticoat with nanocrystalline silver in the management of burns. Colloids and Surface., 47:160-166.

Devina Merin, D., Prakash, S and Valentine Bhimba, B. (2010). Antibacterial screening of silver nanoparticles synthesized by marine micro algae. Asian Pacific Journal of Tropical Medicine., 3: 797-799.

Fortin, D. and Beveridge, T.J. (2000). Mechanistic routes towards biomineral surface development. In: Biomineralisation: From Biology to Biotechnology and Medical Application (E. Baeuerlein Ed.), Wiley-VCH, Verlag, Germany, 294pp.

Ganesh Babu, M. Sridhar, J. Gunasekaran, P. (2011). Global transcriptome analysis of Bacillus cereus ATCC 14579 in response to silver nitrate stress .Journal of Nanobiotechnology. 9:49-59.

Huang, J.L, Li ,Q.B, Sun, D.H, Lu, Y.H, Su, Y.B, Yang, X. Wang, H.X, Wang, Y.P, Ingle, A. Gade, A. Pierrat, S. Sonnichsen,C. Rai, M. (2008). Mycosynthesis of silver nanoparticles using the fungus Fusarium acuminatum and its activity against some human pathogenic bacteria.Current Nanoscience. 4: 141-144.

Ip, M, Lui, S.L, Poon, V. K, Lung, I. Burd, A. (2006). Antimicrobial activities of silver dressings: an in vitro comparison. J. Medical Microbiology., 55:59-63.

Jeeva P. Ramya, K and Edith Rena, A. (2012). Extracellular biosynthisis of silver nanoparticles by culture supernatant of Pseudomonas aeruginose. Indian journal of Biotechnology. 11:72-76.

Kathiresan, K. Manivannan, S. Nabeel, M. A. and Dhivya, B. (2009). Studies on silver nanoparticles synthesized by a marine fungus, Penicillium fellutanum isolated from coastal mangrove sediment. Colloidal and Surface, 71(B):133-137.

Klaus, T. Joerger, R. Olsson, E. and Granqvist, C.G. (1999). Silver-based crystalline nanoparticles,microbially fabricated. Proceddings of the Natlional Acadamy of Sciences of the United States., 96:13611-13614.

Mohanpuria, P. Rana, N.K and Yadav, S.K. (2008). Biosynthesis of nanoparticles: Technological concepts and future applications. Journal of Nanoparticles Research., 10: 507-517.

Mubarak Ali, M. Sasikala, M. Gunasekaran, N. Thajuddin, (2011). Biosynthesis and characterization of silver nanoparticles using marine cyanobacterium, Oscillatoria willei . Digest J. Nanomaterials and Biostructures. 6: 385-390.

Petica, A. Gavriliu, S. Lungu, M. Buruntea, N and Panzaru, C. (2008). Colloidal silver solutions with antimicrobial properties. Material Science and Engineering. 152:22-27.

Rai, M Yadav, A and Gade A. (2009) Silver nanoparticles as a new generation of antimicrobials. Biotechnology Advances.27:76-83.

Russell, A. D and Hugo, W. B. (1994). Antimicrobial activity and action of silver. Progress in Medical Chemistry. 31:351-370.

Saifuddin, N .Wong, C. W and Nuryasumira, A.A. (2009). Rapid biosynthesis of silver nanoparticles using culture supernatant of bacteria with microwave irradiation. Eur Journal of Chemistry.6: 6170.

Sathyavathi, R., Balamurali Krishna, M., Venugopal Rao, S., Saritha, R., arayana Rao, D. (2010.) Biosynthesis of silver nanoparticles using Coriandrum Sativum leaf extract and their application in nonlinear optics. Advanced Science Letters., 3: 1-6.

Selvakumar, D. (2010). Marine Streptomyces as a novel source of bioactive ubstances. World. J. Microbiol. Biotechnol., 26:2123-2139. 
Shankar, S. S., Rai A, Ankamwar, B., Singh, A., Ahmad A. Sastry, M. (2004). Biological synthesis of triangular gold nanoprisms. Nat. Mater., 3: 482-488.

Shirley, A. Dayanand, B. D, Sreedhar, Band Dastager, G. (2010). Antimicrobialactivity of silver nanoparticles synthesized from novel streptomyces species. Digest J. Nanoma.\& Biostructures. 5(2): 447 - 451.

Sondi,I and Salopek-Sondi, B. (2004). Silver nanoparticles as antimicrobial agent: a case study on E. coli as a model for Gram negative bacteria. Journal of Colloid and Interface Science., 275: 177-179.

Takizawa, M., Colwell, R.R and Lill, I. (1993). Isolation and diversity of actinomycetes in the Chesapeake Bay. Applied j. Environ. Microbiology., 59: 997-1002.

Vijayaraghavan, R., Krishna Prabha, V. and Rajendran S. (2012). Biosynthesis of silver nanoparticles by a marine bacterium Bacillus subtilis strain and its antifungal effect. World Journal of Science and Technology., 2:01-03

Vivek, M., Senthil Kumar, P., Steffi, S., Sudha, S. (2011). Biogenic silver nanoparticles by Gelidiella acerosa extract and their antifungal effects. Avicenna Journal of Medical Biotechnology.3143-148.

Ymonier, C. A., Scholotterbeck, U., Antonietti, L., Zacharias, P., Thomann, R., Iller, J.C.T., Mecking, S. (2002). Hybrids of silvernanoparticles with amphiphilichyperbranched macromolecules exhibiting antimicrobial properties. Chemical Communications., 24: 3018-3020. 